\title{
PROTOCOLO DE AVALIAÇÃO DO NÍVEL DE FLEXIBILIDADE DOS ISQUIOTIBIAIS POR FOTOGRAMETRIA
}

\author{
Andrea Perin \\ Mestre pelo Programa de Pós-Graduação em Engenharia Biomédica, da Universidade \\ Tecnológica Federal do Paraná. E-mail: andreaperin@terra.com.br \\ Eduardo Borba Neves \\ Doutor em Engenharia Biomédica pela Universidade Federal do Rio de Janeiro, Doutor em \\ Saúde Pública e Meio Ambiente pela Escola Nacional de Saúde Pública (FIOCRUZ), \\ Docente do Programa de Pós-Graduação em Engenharia Biomédica da Universidade \\ Tecnológica Federal do Paraná. E-mail: borbaneves@hotmail.com

\section{Leandra Ulbricht} \\ Doutora em Engenharia de Produção pela Universidade Federal de Santa Catarina, Pós- \\ Doutorado no Departamento de Saúde Comunitária da Universidade Federal do Paraná, \\ Docente do Programa de Pós-Graduação em Engenharia Biomédica da Universidade \\ Tecnológica Federal do Paraná. E-mail: leandraulbricht@utfpr.edu.br
}

\section{RESUMO}

O teste de sentar e alcançar (TSA) é o teste mais comum para se avaliar a flexibilidade dos isquiotibiais. Apesar de muito utilizado, alguns estudos contestam sua utilização devido a fatores que podem mascarar o seu resultado como, por exemplo, a diferença de proporção de comprimento entre os membros inferiores e superiores, a flexibilidade da coluna vertebral e a abdução escapular. Algumas medidas realizadas em imagens permitem determinar posições relativas entre pontos de referências, ângulos, distâncias e áreas. Neste sentido, o objetivo deste estudo foi desenvolver um protocolo de avaliação do nível de flexão do quadril (alongamento dos isquiotibiais), flexão da coluna lombar e da coluna torácica, por meio da fotogrametria, na posição final do TSA. Para desenvolver o protocolo de avaliação por fotogrametria utilizou-se uma câmera digital da marca Sony ${ }^{\circledR}$ de 10 megapixels de resolução de imagem, um tripé da marca Midas ${ }^{\circledR}$, fita adesiva dupla face, um lápis dermográfico, e três marcadores tipo "cotovelos" de Poliestireno de $90^{\circ}$ da marca Fame ${ }^{\circledR}$. Para a realização da parte experimental do novo protocolo, utilizou-se uma amostra de 30 voluntários de 18 e 19 anos do sexo masculino que assinaram o Termo de Consentimento Livre e Esclarecido. A utilização da análise cinemática angular obtida pela fotogrametria permitiu quantificar o movimento, determinando em valores angulares, a contribuição dos segmentos corporais na execução do TSA. A partir da analise dos resultados, pode-se observar também que o TSA não faz uma avaliação confiável da flexibilidade de isquiotibiais, pois pela homogeneidade dos valores encontrados para o ângulo Fq na amostra estudada, conclui-se que o que fez os sujeitos serem classificados em categorias diferentes (de "fraco" até "excelente") não foi apenas a maior ou menor utilização dos músculos isquiotibiais, mas sim, a soma das contribuições dos segmentos lombar e torácico, pois participam ativamente deste movimento.

PALAVRAS-CHAVE: teste de sentar e alcançar, flexibilidade dos isquiotibiais, fotogrametria, flexão lombar, flexão torácica. 


\title{
ASSESSMENT PROTOCOL OF HAMSTRING FLEXIBILITY LEVEL BY PHOTOGRAMMETRY
}

\begin{abstract}
The sit and reach test (SR) is the most common test to assess hamstring flexibility. Although widely used, some studies deny its use due to factors that can mask the result, for example, the difference in proportion in length between the lower and upper limbs, the flexibility of the spine and scapular abduction. Some images allow measurements in determining the relative positions between reference points, angles, distances and areas. In this sense, the aims of this study was to develop a protocol for assessing the level of hip flexion (hamstring stretching), flexion of the lumbar spine and the thoracic spine, by photogrammetry, at the final position of the SR. To develop the evaluation protocol for photogrammetry used a digital camera from Sony ${ }^{\circledR}$ 10-megapixel image resolution, a tripod brand Midas ${ }^{\circledR}$, double-sided tape, a dermographic pencil, and three markers like "elbows" $\left(90^{\circ}\right)$ of Polystyrene Fame ${ }^{\circledR}$. To perform the experimental part of the new protocol, we used a sample of 30 volunteers aged 18 and 19 males who signed the consent form. The use of kinematic analysis obtained by photogrammetry allowed quantifying the motion, determining angles, the contribution of body segments in the execution of the SR. From the analysis of the results, one can also observe that the SR does not make a reliable assessment of hamstring flexibility because the homogeneity of values for the Fq angle in the sample, it is concluded that what made the subjects were classified in different categories (from "poor" to "excellent") was not only a greater or lesser use of the hamstring muscles, but the sum of the contributions of the lumbar and thoracic segments, for actively participating in this movement.
\end{abstract}

KEYWORDS: sit and reach test, hamstring flexibility, photogrammetry, lumbar flexion, thoracic spine flexion.

\section{PROTOCOLO DE AVALIAÇÃO DO NÍVEL DE FLEXIBILIDADE DOS ISQUIOTIBIAIS POR FOTOGRAMETRIA}

\section{INTRODUÇÃO}

Os ossos, ligamentos, músculos, tendões e a pele são limites estruturais da flexibilidade, a qual pode afetar fatores como amplitude de movimento, atividade física e o risco de ocorrer lesões (FERNANDEZ; STUBBS). O nível inapropriado de flexibilidade pode acarretar problemas como a lombalgia. Nos Estados Unidos, estima-se que esse problema contribui, aproximadamente, com a perda de um bilhão de dólares por ano em produtividade e serviços, além de 225 milhões em indenizações trabalhistas. Normalmente, a falta de flexibilidade ocorre principalmente nos músculos posteriores da coxa, região lombar, região anterior do quadril, pescoço e ombros (ALLSEN et al., 2001).

Os músculos posteriores da coxa, também denominados isquiotibiais, são caracterizados como músculos poliarticulares e com diversas funcionalidades, o que lhes propicia uma condição de tendência ao encurtamento muscular (DAVIS et al., 2005). Ayala et al (2012), relatam que avaliar a flexibilidade dos isquiotibiais é imprescindível, pois o nível 
inapropriado de flexibilidade pode causar lesões nos tendões da musculatura, dores lombares, tendinopatia patelar, dano muscular após exercício excêntrico, redução do desempenho atlético, entre outros. Assim, Kiss (2003) complementa afirmando que a avaliação da flexibilidade permite identificar grupos musculares com déficit de comprimento, possibilitando uma intervenção preventiva para evitar prováveis lesões e melhorar o desempenho.

O TSA é o teste mais comum para se avaliar a flexibilidade dos isquiotibiais e está presente em muitas baterias de testes que visam avaliar desempenho atlético e aptidão física relacionada à saúde. Apesar de muito utilizado, alguns estudos contestam sua utilização devido a fatores que podem mascarar o seu resultado como, por exemplo, a diferença de proporção de comprimento entre os membros inferiores e superiores, a flexibilidade da coluna vertebral e a abdução escapular (AYALA; SAINZ DE BARANDA, 2011; CARDOSO et al., 2007; CORNBLEET; WOOLSEY, 1996; HOEGER et al., 1990; LÓPEZ-MIÑARRO et al., 2009; PERIN et al., 2012).

Essas questões motivaram a criação de novos testes e versões modificadas do próprio TSA, entretanto, alguns estudos observaram que essas versões modificadas apresentaram validade semelhante para avaliação de isquiotibiais, porém nenhuma relação com a avaliação de flexibilidade lombar. O que permitiu concluir que os testes são semelhantes, apresentando apenas algumas variações metodológicas (BALTACI et al., 2003; CASTROPIÑERO et al., 2009; CHUNG; YUEN, 1999; DANNY et al., 1998; JACKSON; BAKER, 1986; LEMMINK et al., 2003; LIEMOHN et al., 1994; MIYAZAKI et al., 2010; PATTERSON et al., 1996).

De acordo com López-Miñarro (2007), o movimento realizado para executar o TSA envolve a flexão do quadril, da coluna lombar e torácica. Entretanto, não foi estabelecida até o presente momento uma versão modificada do TSA capaz de identificar de que maneira os diferentes segmentos da coluna vertebral atuam no movimento. Ou seja, tornase necessário a elaboração de um teste que analise e quantifique como o encurtamento ou a flexibilidade da coluna torácica, lombar e quadris podem influenciar e alterar o movimento através de mecanismos de compensação.

Uma das maneiras que possibilitam essa avaliação é utilizar a análise cinemática angular por meio da fotogrametria. A fotogrametria consiste em uma técnica que registra, mede e interpreta imagens fotográficas de objetos, meio ambiente ou seres humanos. As medidas realizadas nas imagens interligam-se a outras fontes informativas que detêm ferramentas para determinar posições relativas entre pontos de referências, ângulos, distâncias e áreas (RICIERI, 2005). Neste sentido, o objetivo deste estudo foi desenvolver um protocolo de avaliação do nível de flexão do quadril (alongamento dos isquiotibiais), flexão da coluna lombar e da coluna torácica, por meio da fotogrametria, na posição final do Teste de Sentar e Alcançar (TSA).

\section{MATERIAIS E MÉTODOS}

Para desenvolver o protocolo de avaliação por fotogrametria utilizou-se uma câmera digital da marca Sony ${ }^{\circledR}$ de 10 megapixels de resolução de imagem, um tripé da marca Midas ${ }^{\circledR}$, uma balança digital da marca Tanita ${ }^{\circledR}$, duas fitas métricas, fita adesiva, fita adesiva dupla 
face, um lápis dermográfico, marcadores adesivos circulares brancos da marca Pimaco ${ }^{\circledR}$, com 9 milímetros de diâmetro e três marcadores tipo "cotovelos" de Poliestireno de $90^{\circ}$ da marca Fame ${ }^{\circledR}$.

Esses cotovelos são utilizados na construção civil para proteger fiações elétricas. Entretanto, por apresentarem algumas características como peso desprezível, boa aderência à pele e fácil reconhecimento na imagem, foram selecionados para serem utilizados como marcadores proeminentes de fixação na coluna vertebral.

Além disso, utilizou-se um banco padrão da marca Terrazul ${ }^{\circledR}$ para medição de amplitude de alongamento da região posterior do tronco e músculos posteriores da coxa, denominado popularmente como "Banco de Wells". Esse banco é feito de madeira de 31 centímetros de altura, 64,5 centímetros de comprimento e 40 centímetros de largura. Em sua base superior há uma escala métrica de 50 centímetros com um dispositivo móvel deslizante que permite medir o alcance do avaliado.

Para a realização da parte experimental do novo protocolo, utilizou-se uma amostra de 30 voluntários de 18 e 19 anos do sexo masculino que assinaram o Termo de Consentimento Livre e Esclarecido. O presente estudo atendeu a todas as exigências da Resolução 196/96 do Conselho Nacional de Saúde e teve seu protocolo aprovado no Comitê de Ética em Pesquisa do Centro Universitário Campos Andrade (Uniandrade) sob o número 422.

Para a identificação dos ângulos, as imagens captadas foram importadas para o software de livre acesso denominado ImageJ 1.4. Os valores dos ângulos foram planilhados e analisados pelo software SPSS versão 20, o qual gerou a estatística descritiva, teste de Kolmogorov-Smirnov e análise de variância (ANOVA), com o com Fisher's least significant difference (LSD) como teste post hoc. O nível de significância utilizado foi de $p$ $<0,05$.

\section{RESULTADOS}

Os resultados estão organizados em duas seções. A primeira diz respeito à elaboração do protocolo de avaliação, a qual descreve em detalhes os passos e procedimentos adotados para a concepção e definição do protocolo. A segunda sessão faz referência aos resultados dos dados obtidos pela aplicação experimental do referido protocolo, os quais possibilitaram analisar a sua eficiência.

\subsection{DESENVOLVIMENTO DO PROTOCOLO DE AVALIAÇÃO}

Por meio da técnica de anatomia palpatória, identificou-se a quinta vértebra lombar, décima segunda vértebra torácica e sétima vértebra cervical, todas sinalizadas com os marcadores esféricos de nove milímetros.

Foi solicitado ao indivíduo que, em pé, fizesse uma flexão de tronco para a colocação dos marcadores proeminentes tipo "cotovelos". A flexão de tronco para a colocação dos marcadores era necessária para que eles seguissem o mesmo padrão da linha das curvaturas fisiológicas da coluna vertebral. 
Esses cotovelos foram selecionados porque atenderam alguns requisitos necessários para serem aderidos a superfície da pele na região dorsal, ou seja, tinham fácil reconhecimento na imagem digital, peso desprezível, formato semelhante à letra "L" com angulação de $90^{\circ}$ e hastes de quatro centímetros de comprimento (figura 1).

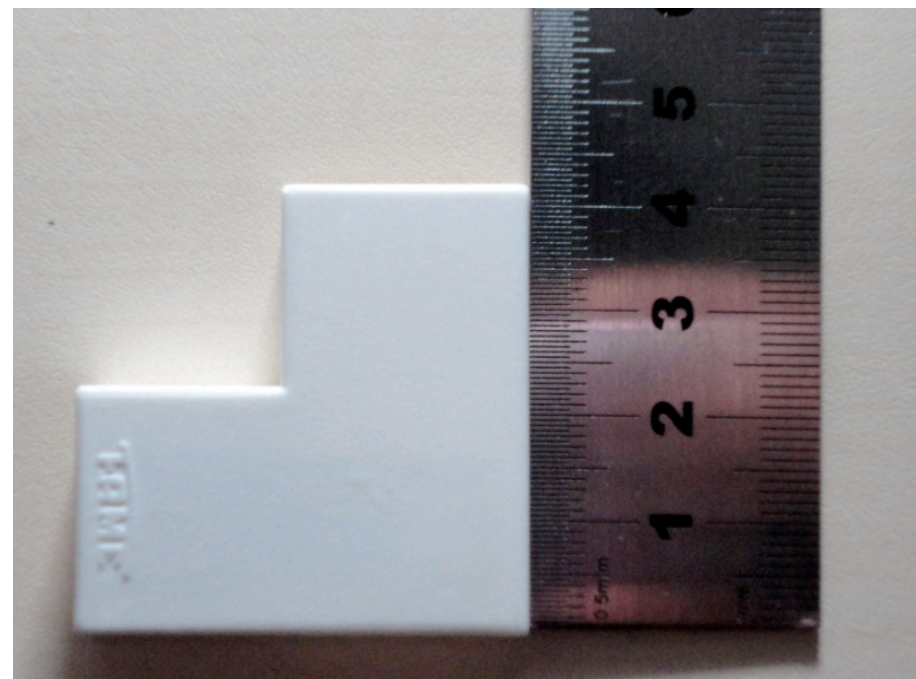

Figura 1 - Marcador proeminente tipo "cotovelo" da marca Fame ${ }^{\circledR}$

Assim uma das hastes do primeiro marcador era colada, por meio de fita adesiva dupla face, na região correspondente ao corpo da quinta vértebra lombar. Da mesma forma, o segundo marcador era colocado na da décima segunda vértebra torácica e o terceiro marcador na sétima vértebra cervical (figura 2).

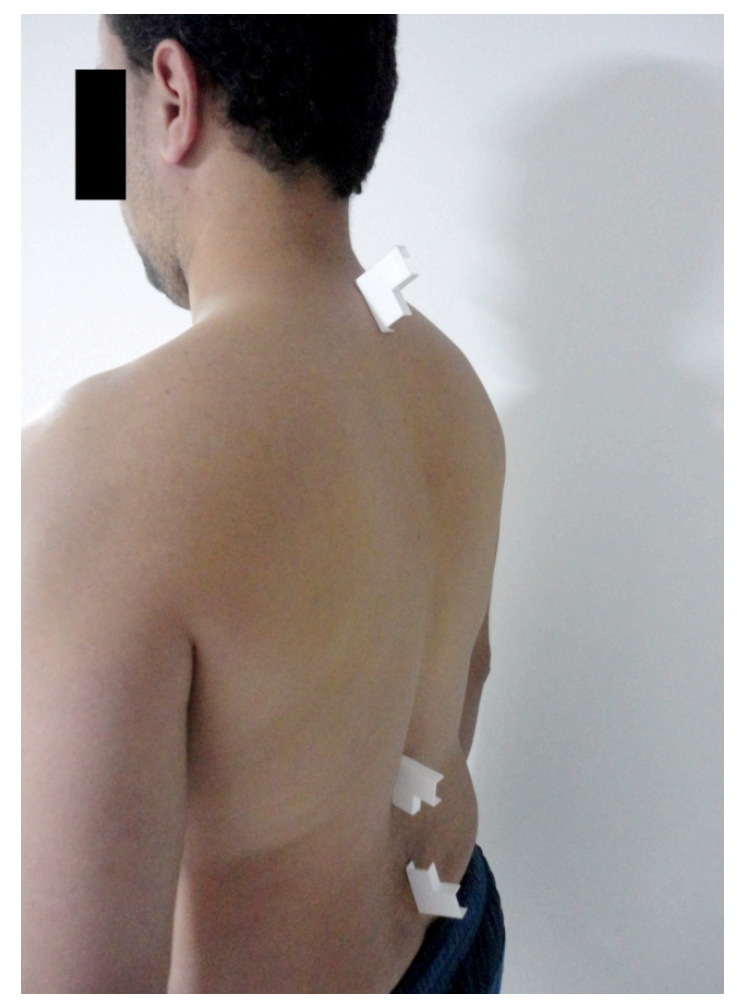

Figura 2 - Marcadores proeminentes fixados na coluna vertebral: sétima vértebra cervical, décima segunda vértebra torácica e quinta vértebra lombar 
Depois das marcações, o voluntário era orientado a fazer o teste de Sentar e Alcançar. Na posição inicial, ele deveria sentar-se de frente para o banco, com as pernas estendidas e encostando a região plantar de seus pés na base de madeira que fica logo abaixo do platô superior, onde se localiza a escala métrica. O tronco deveria permanecer ereto, braços estendidos, uma mão sobre a outra e pontas dos dedos encostadas no dispositivo móvel da escala. Depois, o indivíduo deveria empurrar esse dispositivo móvel o máximo que conseguisse, sem dar dois toques ou fazer um movimento brusco (POLLOCK; WILMORE, 1993). Se as pernas flexionassem durante o movimento ou o dispositivo fosse empurrado indevidamente, repetia-se o teste. Foram captadas, por meio de fotografia, imagens da posição final do teste.

A distância entre a câmera fotográfica e o indivíduo e a altura do tripé foram mensuradas com fita métrica e demarcadas, essa medida foi padronizada e todos os avaliados foram fotografados com a mesma distância.

O método utilizado para a avaliação das imagens foi a Fotogrametria, a qual consiste em se aplicar a fotografia para deduzir dimensões de objetos em uma imagem. Ou seja, é uma técnica que permite medir um objeto tanto em relação as suas formas quanto situação espacial, por meio de registro fotográfico (RICIERI, 2005).

As imagens captadas foram importadas para o software de livre acesso denominado ImageJ 1.4, que possibilitou o ajuste da imagem em relação ao plano horizontal, e a medição dos ângulos através das ferramentas denominadas "straight" e "angle tool".

Diante disso, cinco ângulos foram criados para identificar a participação dos diferentes segmentos da coluna vertebral no movimento de flexão do tronco obtido através do teste de Sentar e Alcançar. O primeiro ângulo criado foi denominado de "Fqlt", ou seja, uma inferência ao ângulo da flexão total do tronco, o qual englobou a região do quadril, região lombar e torácica. Para medi-lo era necessário traçar uma linha em cima da haste do marcador que fazia a projeção da localização da sétima vértebra cervical e traçar outra linha perpendicular a esta que deveria estar a $90^{\circ}$ em relação ao plano vertical (Figura 3).

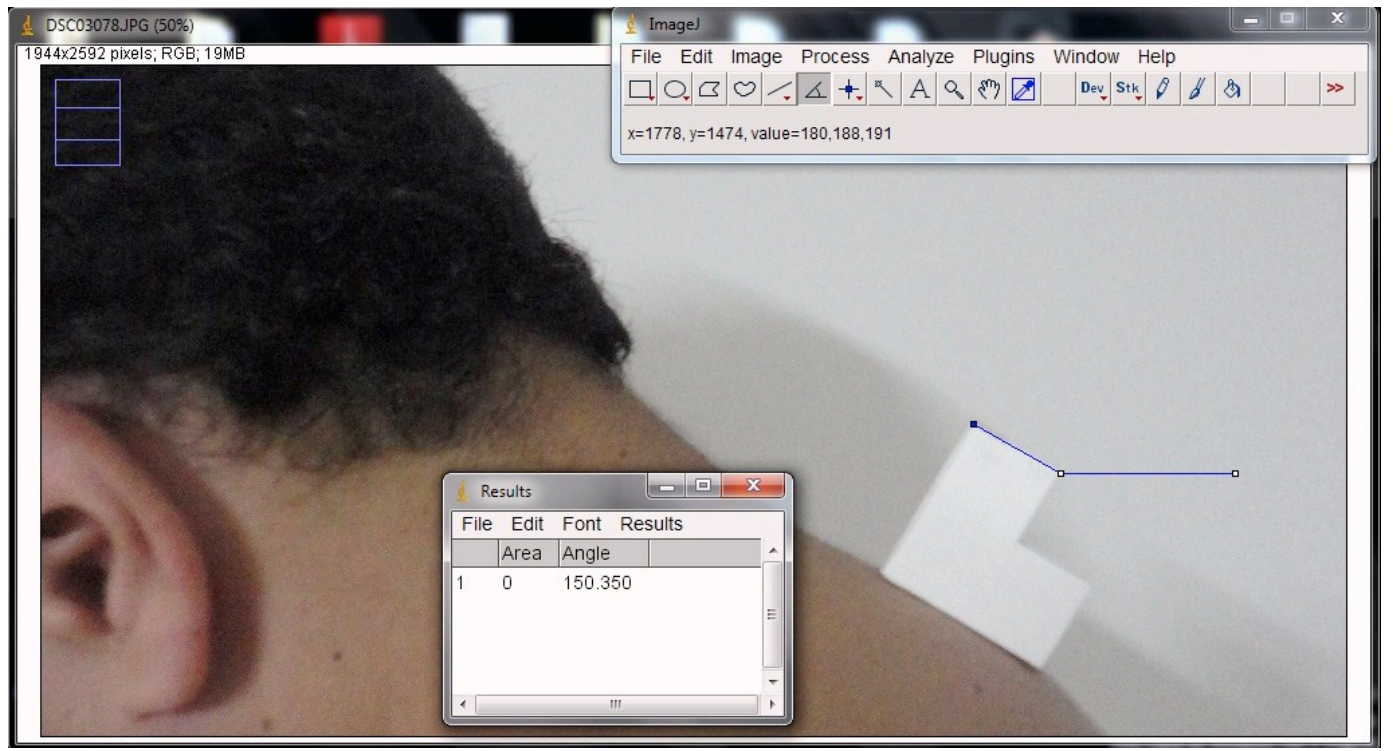

Figura 3 - Medição do ângulo Fqlt no ImageJ 
O segundo ângulo, foi o "Fql" que tinha como objetivo fazer uma inferência ao ângulo de flexão do quadril e da lombar. Assim como o primeiro ângulo, neste traçava-se uma linha em cima da haste do marcador que estava em cima do platô da décima segunda vértebra torácica e outra linha perpendicular a $90^{\circ}$ graus do plano vertical.

E o terceiro ângulo, "Fq", identificava apenas a flexão do quadril. Para forma-lo, também se utilizava a linha que passava rente ao marcador com outra linha perpendicular de $90^{\circ}$. $\mathrm{O}$ ângulo $\mathrm{Fq}$ representou o alongamento dos músculos flexores do quadril, os quais são situados na parte posterior da coxa e da região glútea. São eles: glúteo máximo, fibras posteriores do glúteo médio, fibras posteriores do glúteo mínimo, porção extensora do adutor magno e, em principal, os músculos bíceps femoral, semitendinoso e semimembranoso (FLOYD; THOMPSON, 2002).

Já o ângulo Fql além de representar também o alongamento dos músculos flexores do quadril, proporcionou o alongamento das fibras dos músculos eretores da espinha, mais especificadamente, a porção denominada iliocostal lombar e a porção lombar dos paravertebrais. E o ângulo Fqlt, agregou a magnitude do alongamento dos músculos flexores do quadril, paravertebrais e eretores da espinha - ilicostais, longuíssimo e espinhais (RASCH, 2008). Partindo desses três ângulos principais, criou-se o "Fl" (ângulo de flexão lombar) que se originou da subtração do "Fql" pelo "Fq", além do "Ft" (ângulo da flexão torácica), o qual era a diferença da subtração do "Fqlt" pelo "Fql" (figura 4).

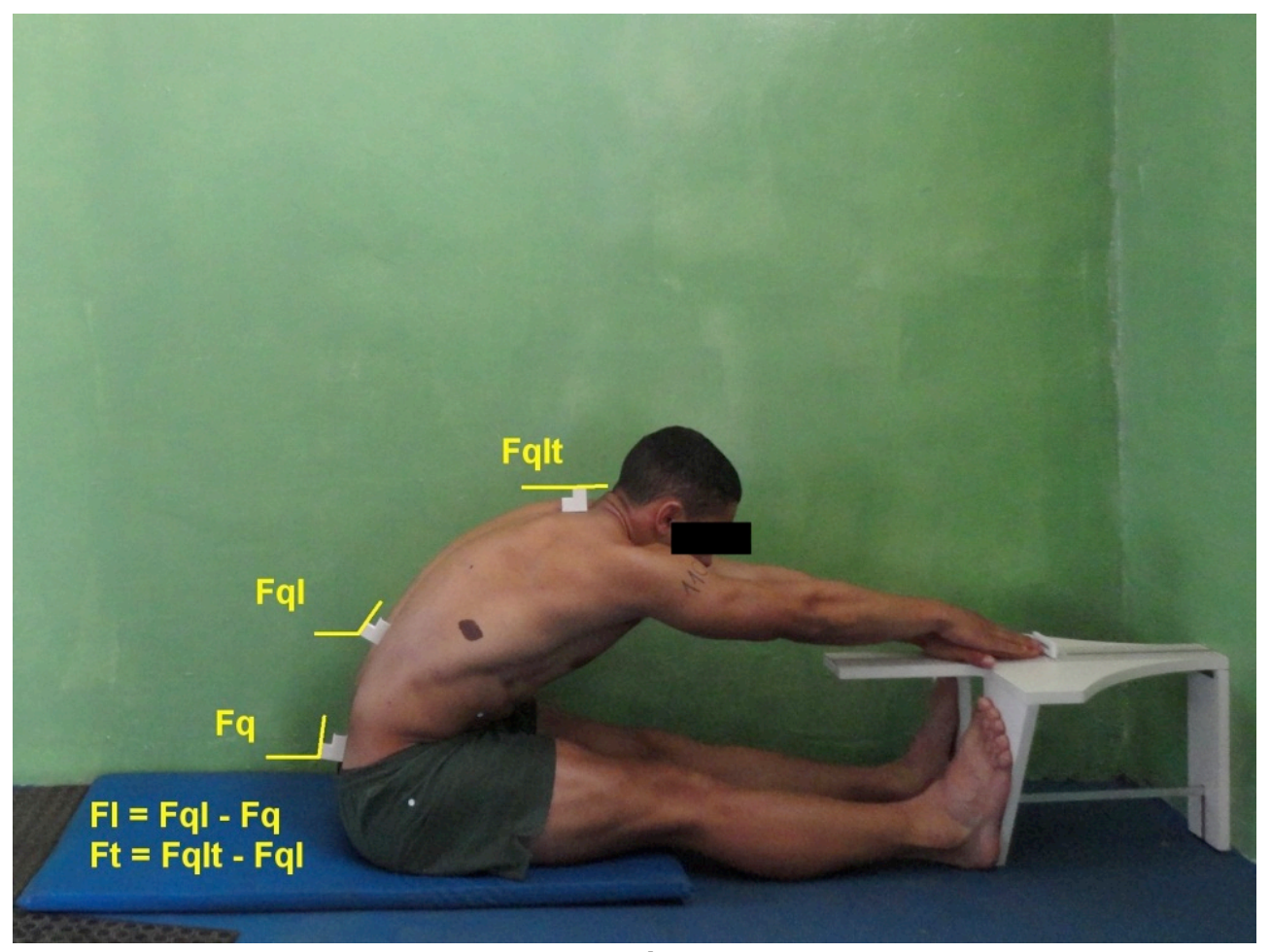

Figura 4 - Execução do TSA com os ângulos formados pelos marcadores

Para identificar o percentual de contribuição de cada segmento para a flexão total de tronco, dividiu-se, primeiramente, o Fq pelo Fqlt e seu quociente foi multiplicado por 100, resultando no percentual do quadril (Equação 1). Depois o mesmo foi feito com o quociente da divisão do Fl pelo Fqlt, encontrando o percentual da região lombar (Equação 2) e, por último, obteve-se o percentual da região torácica com a divisão do Ft pelo Fqlt também com o seu quociente multiplicado por 100 (Equação 3). 
PercQuadril $=(\mathbf{F q} /$ Fqlt $) .100$

Equação (1)

PercLombar $=($ Fl $/$ Fqlt $) .100$

Equação (2)

PercTorácica $=($ Ft $/$ Fqlt $) .100$

Equação (3)

\subsection{EXPERIMENTAÇÃO DO MÉTODO}

Para o TSA existe um padrão de referência criado por Pollock e Wilmore (1993), o qual classifica o resultado do TSA em cinco categorias: Fraco (11 cm ou menos); Regular (12 a $13 \mathrm{~cm}$ ); Médio (14 a $18 \mathrm{~cm})$; Bom $(19 \mathrm{a} 21 \mathrm{~cm})$ e Excelente $(22 \mathrm{~cm}$ ou mais). Foi realizado a aplicação do protocolo na amostra, e os dados angulares obtidos de Fq, Fl e Ft foram distribuídos entre as categorias de classificação a partir do resultado do TSA. Assim, as figuras 5, 6 e 7 apresentam os valores dos ângulos Fq, Fl e Ft para cada categoria do TSA.

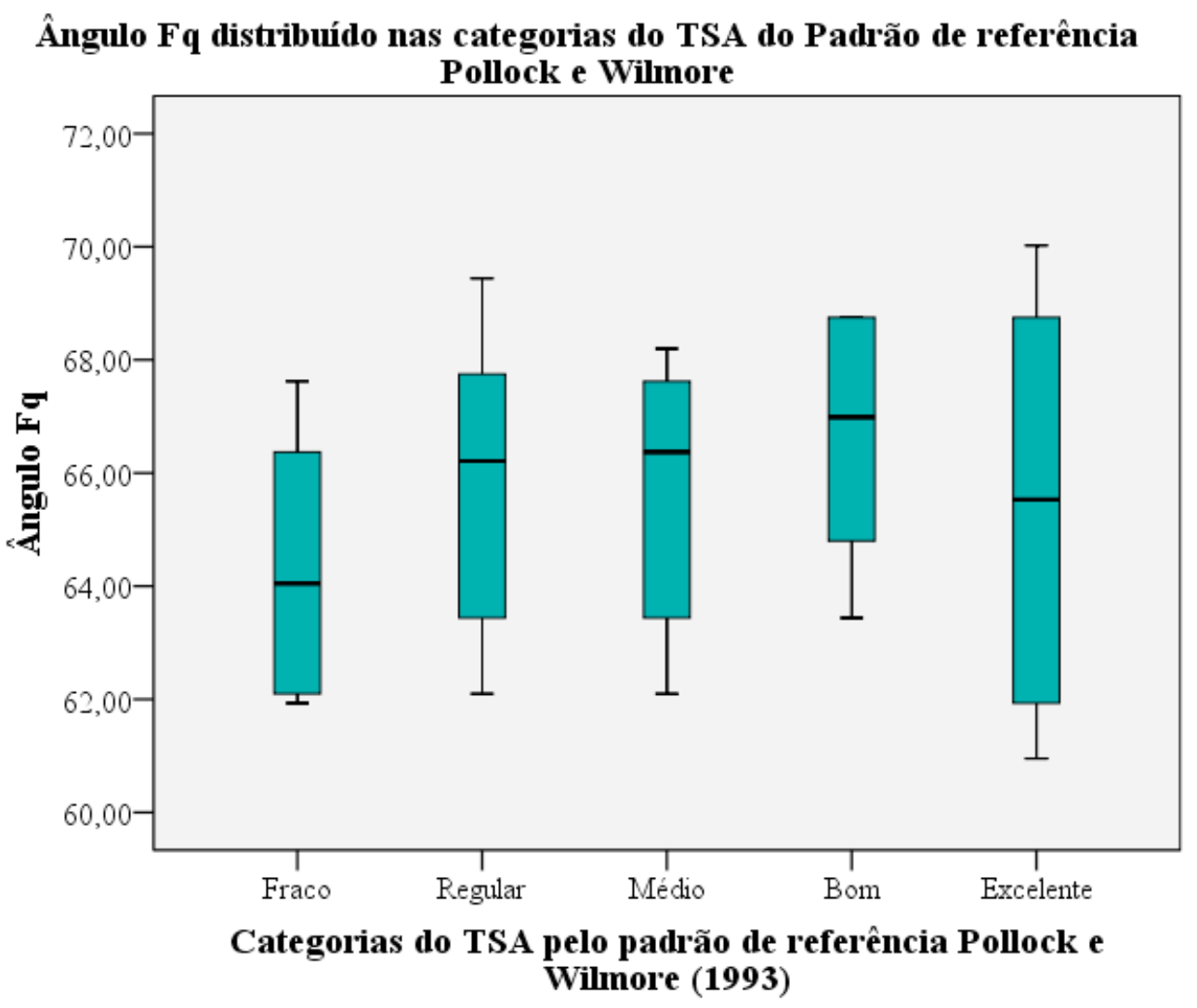

Figura 5. Gráfico do Fq de acordo com as classificações do padrão de referência Pollock e Wilmore (1993) 


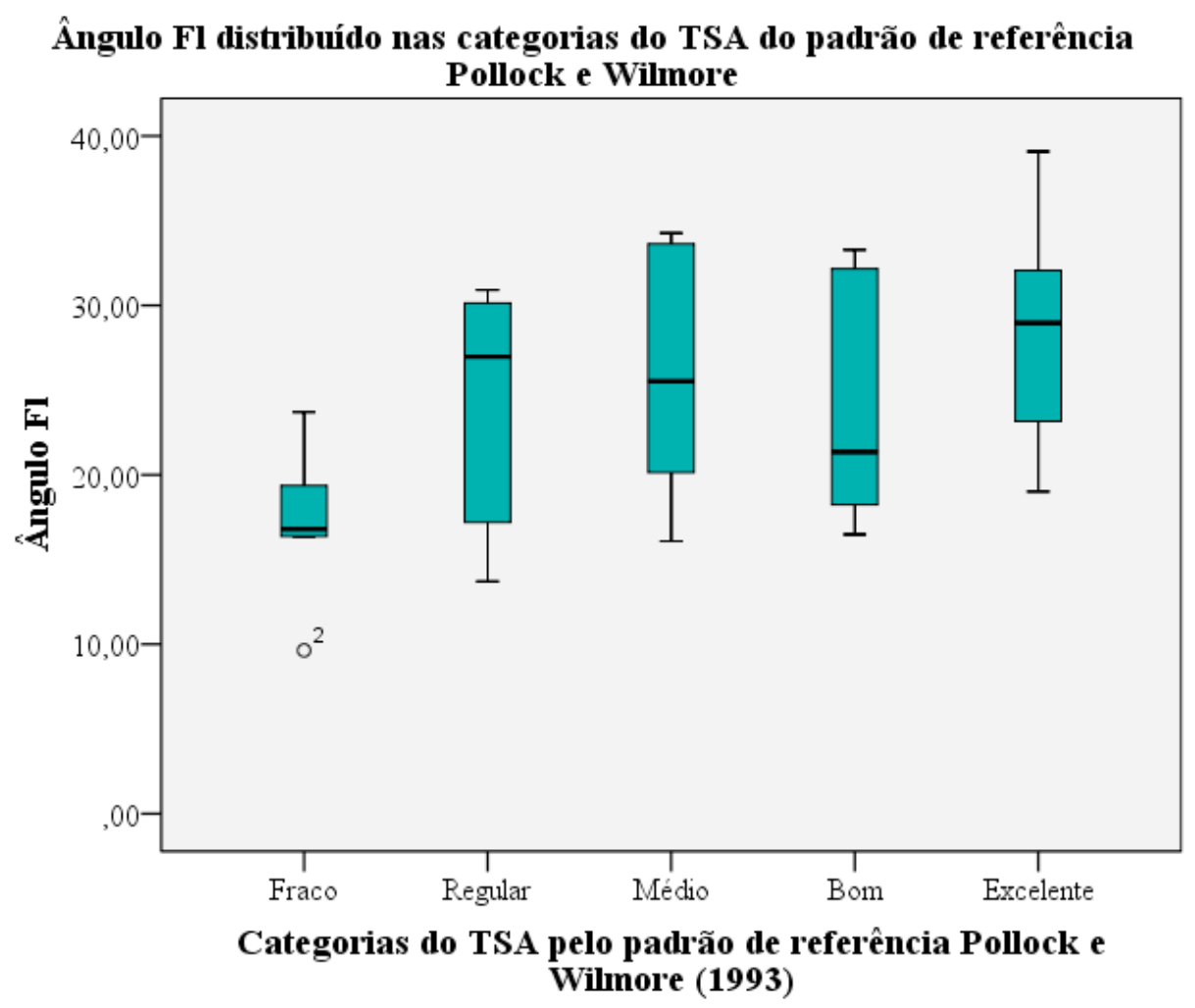

Figura 6. Gráfico do Fl de acordo com as classificações do padrão de referência Pollock e Wilmore (1993)

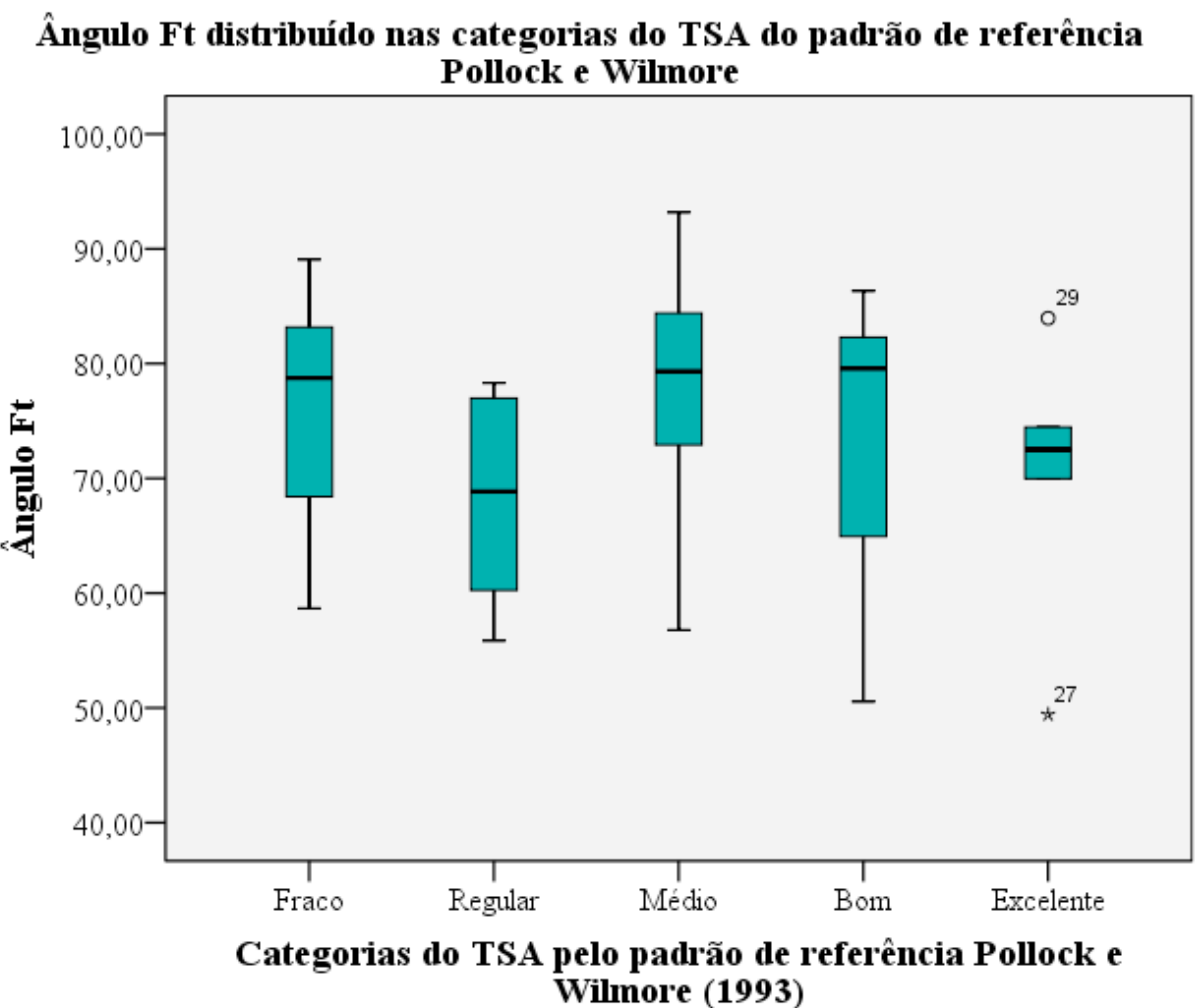

Figura 7. Gráfico do Ft de acordo com as classificações do padrão de referência Pollock e Wilmore (1993) 
A tabela 1 mostra as diferenças significativas entre as categorias do padrão de referência de Pollock e Wilmore para as variáveis Fq, Fl, Ft, e TSA. Entre as categorias de Fq e Ft não houve diferenças significativas entre as médias indicando que a variação desses ângulos, estatisticamente, é muito próxima. Ou seja, não há diferenças consideráveis de flexão de quadril e flexão torácica quando se compara as classificações.

Tabela 1. Diferenças significativas entre as categorias do padrão de referência Pollock e Wilmore das variáveis avaliadas

\begin{tabular}{|c|c|c|}
\hline Variáveis & \multicolumn{2}{|c|}{$\begin{array}{c}\text { Pares com diferenças significativas }(p<0,05) \text { na } \\
\text { ANOVA com post hoc LSD }\end{array}$} \\
\hline $\mathbf{F q}$ & & - \\
\hline Fl & \multicolumn{2}{|c|}{$\begin{array}{l}\text { F e M }\left(-8,75167^{*}\right) \\
\text { F e E }\left(-11,43333^{*}\right)\end{array}$} \\
\hline Ft & & - \\
\hline TSA & $\begin{array}{l}\text { F e R }\left(-4,83333^{*}\right) \\
\text { F e M }\left(-7,66667^{*}\right) \\
\text { F e B }\left(-12,166667^{*}\right) \\
\text { F e E }\left(-16,333333^{*}\right) \\
\text { R e M }\left(-2,833333^{*}\right)\end{array}$ & $\begin{array}{l}\text { Re B }\left(-7,333333^{*}\right) \\
\text { Re E }\left(-11,500000^{*}\right) \\
\text { M e B }\left(-4,500000^{*}\right) \\
\text { M e E }\left(-8,666667^{*}\right) \\
\text { B e E }\left(-4,166667^{*}\right)\end{array}$ \\
\hline
\end{tabular}

Onde: $\mathrm{F}=$ fraco; $\mathrm{R}=$ regular; $\mathrm{M}=$ média; $\mathrm{B}=$ bom; $\mathrm{E}=$ excelente

\section{DISCUSSÃO}

Em relação aos valores angulares, observou-se que o ângulo $\mathrm{Fq}$ obteve uma variação de, aproximadamente, 60 a 70 graus. Já para o ângulo Ft a variação foi de, aproximadamente, 50 a 90 graus. De acordoAcademy of Orthopaedic (1965), a amplitude para o ângulo de flexão do quadril varia de $0^{\circ}$ a $120^{\circ}$ e para flexão torácica de $0^{\circ}$ a $80^{\circ}$. E Boone e Azen (1979), em seu estudo realizado com 109 homens com idade entre 1,5 anos a 54 anos, encontraram que a média de flexão de quadril para 53 homens que tinham 19 anos, ou menos, foi de $123,4^{\circ}$.

López-Miñarro et al (2009), avaliou os ângulos de flexão de quadril, torácica e lombar com um inclinômetro no movimento de flexão do tronco durante o TSA e obteve ângulos de $105^{\circ}, 70^{\circ}$ e $28^{\circ}$, respectivamente. Em outro estudo, López-Miñarro et al (2007), obteve resultados semelhantes para flexão de coluna torácica e coluna lombar, aproximadamente $70^{\circ}$ e $30^{\circ}$, respectivamente. No mesmo sentido, Kendall et al (2007), afirmaram que o comprimento normal dos isquiotibiais permite que a pelve faça uma flexão em direção às coxas de maneira que o sacro forme um ângulo de $80^{\circ}$ com o eixo horizontal.

Analisando a figura 5 referente ao gráfico do ângulo $\mathrm{Fq}$ distribuído nas categorias do padrão de referência proposto, nota-se que possui uma pequena variação nos dados que fazem inferência ao ângulo de flexão do quadril, ou seja, mesmo em categorias melhores, o Fq que diz respeito diretamente ao nível de flexibilidade dos isquiotibiais não obteve valores muito maiores do que em categorias consideradas ruins. 
A figura 6 mostra o gráfico do Fl também distribuído nas categorias do padrão de referência de Pollock e Wilmore, e é possível observar que o seu valor aumenta à medida que as categorias progridem, indicando que nas melhores classificações ocorre uma influência considerável da coluna lombar podendo estar relacionado ao ato de compensação do movimento.

Alter (1991), explica que a postura considerada ideal para o alongamento dos isquiotibiais é manter uma inclinação pélvica anterior e o dorso superior estendido quando o tronco movimenta-se em direção às coxas. Ou seja, deve-se existir uma linha reta entre o sacro e a parte superior da cabeça, pois assim a tuberosidade isquiática (origem dos isquiotibiais) é colocada em uma posição mais distante das inserções tibial proximal e fibular, fazendo com que a tensão das fibras musculares seja aumentada.

Entretanto, o autor relata que quando existe o encurtamento, o corpo tende a fazer uma retroversão da pelve além da formação de uma curva acentuada na região dorsal. Essa compensação é proveniente dos padrões de flexão combinados das vértebras cervicais, torácicas e lombares. Com isso, a inclinação pélvica posterior aproxima a origem dos isquiotibiais à sua inserção e o arredondamento na região dorsal impede que ocorram mais deslocamentos angulares e lineares o que exige menos amplitude de movimento (ALTER, 1991).

Em relação ao ângulo Ft (Figura 7) distribuído nas categorias propostas por Pollock e Wilmore, pode-se observar que entre as categorias existe uma grande variabilidade da participação da coluna torácica no movimento, o que também indica a influência que essa articulação exerce no movimento.

Assim, pelo fato do TSA considerar apenas o valor obtido no banco de Wells, ele acaba ignorando o comportamento dos segmentos corporais que influenciam o movimento de flexão do tronco, pois considera apenas a amplitude total. Assim, apenas pela pontuação obtida e pelos padrões de referência já estabelecidos, não é possível predizer com propriedade o nível flexibilidade de músculos posteriores de coxa.

O ângulo Ft obteve diferença significativa apenas em dois pares de categorias, já para a variável TSA todos os pares de categorias foram indicados como diferentes estatisticamente. Esse fato evidencia que por mais que os valores do TSA possam se mostrar significativamente diferentes, os dados angulares, representantes da real participação dos segmentos corporais, não podem ser analisados a partir desse padrão de referência, pois o mesmo não faz a diferenciação correta dos valores que seja capaz de identificar presença ou ausência de encurtamento muscular, bem como compensações de musculaturas acessórias na execução do movimento.

Por esse motivo torna-se extremamente difícil avaliar isoladamente a flexibilidade dos isquiotibiais através da flexão de tronco. Pois somente o conjunto de fatores associados como necessidade de se levar em consideração o comportamento dos outros segmentos corporais, a observação padrões de movimento, a quantificação e classificação da relação entre as musculaturas, determinam a real condição dos músculos isquiotibiais, bem como esquema corporal resultante dos diferentes níveis de flexibilidade das regiões participantes do movimento. 


\section{CONCLUSÃO}

A utilização da análise cinemática angular obtida pela fotogrametria permitiu quantificar o movimento, determinando em valores angulares, a contribuição dos segmentos corporais na execução do TSA. A partir da analise dos resultados, pode-se observar também que o TSA não faz uma avaliação confiável da flexibilidade de isquiotibiais, pois pela homogeneidade dos valores encontrados para o ângulo Fq na amostra estudada, conclui-se que o que fez os sujeitos serem classificados em categorias diferentes (de "fraco" até "excelente") não foi apenas a maior ou menor utilização dos músculos isquiotibiais, mas sim, a soma das contribuições dos segmentos lombar e torácico, pois participam ativamente deste movimento.

Assim, recomenda-se que a avaliação da flexão da coluna torácica, da coluna lombar e do quadril (alongamento dos isquiotibiais) seja realizada separadamente, por fotogrametria, com base no protocolo estabelecido neste estudo. Pois desta forma, será possível a determinação da contribuição dos diferentes segmentos corporais, favorecendo a identificação dos mecanismos compensatórios, possibilitando uma intervenção preventiva relacionada à correção postural e melhora do nível de flexibilidade.

\section{REFERÊNCIAS BIBLIOGRÁFICAS}

ALLSEN, P. E.; HARRISON, J. M.; VANCE, B. Exercício e Qualidade de Vida: uma abordagem personalizada. 6. Barueri: Manole, 2001.

ALTER, M. J. Ciência da Flexibilidade. 2. Porto Alegre: Artes Médicas Sul, 1991.

AYALA, F.; SAINZ DE BARANDA, P. Fiabilidad absoluta de las pruebas sit and reach modificado y back saber sit and reach para estimar la flexibilidad isquiosural em jugadores de fútbol sala. Apunts Medicina de L' Esport, v. 46, p. 81-88, 2011.

AYALA, F. et al. Absolute reliability of five clinical tests for assessing hamstring flexibility in professional futsal players. Journal of Science and Medicine in Sport v. 15, p. 142-147, 2012.

BALTACI, G. et al. Comparison of three different sit and reach tests for measurement of hamstring flexibility in female university students. British journal of sports medicine, v. 37, p. 59-61, 2003.

CARDOSO, J. R. et al. Confiabilidade intra e interobservador da análise cinemática angular do quadril durante o teste sentar e alcançar para mensurar o comprimento dos isquiotibiais em estudantes Universitários. Revista Brasileira de Fisioterapia v. 11, p. 133-138, 2007.

CASTRO-PIÑERO, J. et al. Criterion-Related Validity of Sit-and-Reach and Modified Sitand-Reach Test for Estimating Hamstring Flexibility in Children and Adolescents Aged 6 - 17 Years. International Journal of Sports Medicine, v. 30, p. 658-662, 2009. 
CHUNG, P. K.; YUEN, C. K. Criterion-related validity of sit-and-reach tests in university men in Hong Kong. Perceptual and motor skills, v. 88, p. 304-316

1999.

CORNBLEET, S. L.; WOOLSEY, N. B. Assessment of Hamstring Muscle Length in School-aged Children Using the Sit-and-Reach Test and the Inclinometer Measure of Hip Joint Angle. Physical Therapy in Sport, v. 76, p. 850-855, 1996.

DANNY, P. et al. Emg activities between sit-and-reach and stand-and-reach: a pilot study. Proceedings of the 20thAnnual International Conference of the IEEE Engineering in Medicine and Biology Society. 20: 2448-2451 p. 1998.

DAVIS, D. S. et al. The effectiveness of 3 stretching techniques on hamstring flexibility using consistent stretching parameters. Journal of Strength and Conditioning Research, v. 19, n. 1, p. 27-32, 2005.

FERNANDEZ, J. E.; STUBBS, N. B. Mathematical modeling and testing of the sit and reach test. International Journal of Industrial Ergonomics, v. 3, p. 201-205, 1989.

FLOYD, R. T.; THOMPSON, C. W. Manual de Cinesiologia Estrutural. 14. Barueri: Manole, 2002.

HOEGER, W. W. K. et al. Comparing the Sit and Reach With the Modified Sit and Reach in Measuring Flexibility in Adolescents. Pediatric Exercise Science, v. 2, p. 156162, 1990.

JACKSON, A. W.; BAKER, A. A. The relationship of the sit and reach test to criterion measures of hamstring and back flexibility in young females. Research Quarterly for Exercise and Sport, v. 57, n. 183-186, 1986.

KISS, M. A. P. D. Esporte e Exercício: avaliação e prescrição. São Paulo: Roca, 2003.

LEMMINK, K. A. P. M. et al. The Validity of the Sit-and-Reach Test and Modified Sitand-Reach Test in Middle-Aged to Older Man and Women. Research Quarterly for Exercise and Sport, v. 74, p. 331-336, 2003.

LIEMOHN, W. P.; SHARPE, G. L.; WASSERMAN, J. Criterion-related validity of the sit-and-reach test. Journal of Strength and Conditioning Research, v. 8, p. 91-94, 1994.

LÓPEZ-MIÑARRO, P. A. et al. A comparison of the spine posture among several sit-andreach test protocols. Journal of Science and Medicine in Sport, v. 10, p. 456-462, 2007.

LÓPEZ-MIÑARRO, P. A.; ANDÚJAR, P. S. B.; R., G. P. L. A comparison of the sit-andreach test and the back-saver sit-and-reach test in university students. Journal of Sports Science and Medicine, v. 8, p. 116-122, 2009.

MIYAZAKI, J. et al. Relationship between the sit-and-reach distance and spinal mobility and straight leg raising range. Rigakuryoho Kagaku, v. 25, p. 683-686, 2010. 
PATTERSON, P. et al. The Validity and Reliability of the Back Saver Sit-and-Reach Test in Middle School Girls and Boys. Research Quarterly for Exercise and Sport, v. 67, p. 448451, 1996.

PERIN, A. et al. Utilização da Biofotogrametria para a avaliação de flexibilidade de tronco. Revista Brasileira de Medicina do Esporte, v. 18, n. 3, p. 176-180, 2012.

POLlOCK, M. L.; WILMORE, J. H. Exercícios na Saúde e na Doença: Avaliação e Prescrição para Prevenção e Reabilitação. 2. Rio de Janeiro: MEDSI, 1993.

RASCH, P. J. Cinesiologia e anatomia aplicada. 7. Rio de Janeiro: Guanabara Koogan, 2008.

RICIERI, D. V. Biofotogrametria: análise cinemática angular dos movimentos. Curitiba: Inspirar, 2005. 